\title{
Gamma ray sources observation with the ARGO-YBJ detector
}

\author{
S. Vernetto on behalf of the ARGO-YBJ collaboration \\ IFSI-INAF Corso Fiume 4, 10133 Torino, Italy \\ Received: 12 November 2010 - Accepted: 17 December 2010 - Published: 9 February 2011
}

\begin{abstract}
In this paper we report on the observations of $\mathrm{TeV}$ gamma ray sources performed by the air shower detector ARGO-YBJ. The objects studied in this work are the blazar Markarian 421 and the extended galactic source MGROJ1908+06, monitored during $\sim 2$ years of operation.

Mrk421 has been detected by ARGO-YBJ with a statistical significance of $\sim 11$ standard deviations. The observed $\mathrm{TeV}$ emission was highly variable, showing large enhancements of the flux during active periods. The study of the spectral behaviour during flares revealed a positive correlation of the hardness with the flux, as already reported in the past by the Whipple telescope, suggesting that this is a long term property of the source.

ARGO-YBJ observed a strong correlation between $\mathrm{TeV}$ gamma rays and the X-ray flux measured by RXTM/ASM and SWIFT/BAT during the whole period, with a time lag compatible with zero, supporting the one-zone SSC model to describe the emission mechanism.

MGROJ1908+06 has been detected by ARGO-YBJ with $\sim 5$ standard deviation of significance. From our data the source appears extended and the measured extension is $\sigma_{\text {ext }}=0.48^{\circ}+0.26$, in agreement with a previous HESS observation. The average flux is in marginal agreement with that reported by MILAGRO, but significantly higher than that obtained by HESS, suggesting a possible flux variability.
\end{abstract}

\section{Introduction}

ARGO-YBJ is a full-coverage air shower array, located at the Yangbajing Cosmic Ray Laboratory (Tibet, China, $4300 \mathrm{~m}$ a.s.l.). The study of gamma ray sources with energies above a few hundred GeVs is one of the main purposes of the experiment.

Correspondence to: S. Vernetto (vernetto@to.infn.it)
The large field of view ( $\sim 2 \mathrm{sr})$ and the high duty cycle allow a continuous monitoring of the sky in the declination band from $-0^{\circ}$ to $70^{\circ}$. In this paper we report on the observations of two bright gamma ray sources, performed during $\sim 2$ years: the blazar Mrk421 and the extended galactic source MGROJ1908+06.

\section{The ARGO-YBJ experiment}

The ARGO-YBJ detector is composed by a central carpet large $\sim 74 \times 78 \mathrm{~m}^{2}$, made of a single layer of Resistive Plate Chambers (RPCs) with $\sim 92 \%$ of active area, sorrounded by a partially $(\sim 20 \%)$ instrumented area up to $\sim 100 \times 110 \mathrm{~m}^{2}$. The apparatus has a modular structure, the basic data acquisition element being a cluster $\left(5.7 \times 7.6 \mathrm{~m}^{2}\right)$, made of 12 RPCs $\left(2.8 \times 1.25 \mathrm{~m}^{2}\right)$. Each chamber is read by 80 strips of $6.75 \times 61.8 \mathrm{~cm}^{2}$ (the spatial pixels), logically organized in 10 independent pads of $55.6 \times 61.8 \mathrm{~cm}^{2}$ which are individually acquired and represent the time pixels of the detector. The full apparatus is made of 153 clusters for a total active surface of $\sim 6600 \mathrm{~m}^{2}$ (Aielli et al., 2006).

The detector is in stable data taking since November 2007 with a trigger rate of $\sim 3.6 \mathrm{kHz}$ and a duty cycle $\geq 85 \%$.

The current trigger system requires a number of particles hitting the central carpet $N_{\text {pad }} \geq 20$. For each event, the location and the arrival time of every detected particle are recorded, allowing the reconstruction of the shower arrival direction.

The angular resolution and the pointing accuracy of the detector have been evaluated by using the Moon shadow, i.e. the deficit of cosmic rays in the Moon direction. ARGOYBJ observes the Moon shadow with a sensitivity of $\sim 10$ standard deviations per month for events with a multiplicity $N_{\text {pad }} \geq 40$ and zenith angle $\theta<50^{\circ}$, corresponding to a proton median energy $E_{p} \sim 1.8 \mathrm{TeV}$ (Di Sciascio et al., 2008).

According to the Moon shadow data, the semi-aperture of the circular area containing $71.5 \%$ of the signal (that

Published by Copernicus Publications on behalf of the Arbeitsgemeinschaft Extraterrestrische Forschung e.V. 


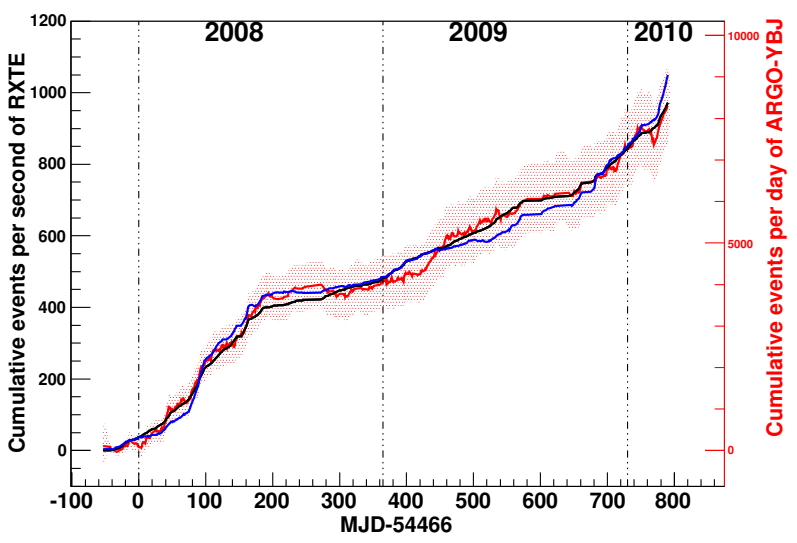

Fig. 1. Mrk421 cumulative light curve. Red line: rate of $\mathrm{TeV}$ gamma rays measured by ARGO-YBJ. The red band indicates the statistical error of one standard deviation. Black line: soft X-rays by RXTE/ASM. Blue line: hard X-rays by Swift/BAT (rescaled in order to have the same total counting rate of RXTE).

maximizes the signal to noise ratio for a Gaussian PSF) is $2.59^{\circ} \pm 0.16^{\circ}, 1.30^{\circ} \pm 0.14^{\circ}$ and $1.04^{\circ} \pm 0.12^{\circ}$ for $N_{\text {pad }} \geq 40$, 100 and 300 , respectively, in agreement with expectations from Monte Carlo simulations.

This angular resolution refers to cosmic ray-induced air showers. The angular resolution for $\gamma$-induced events has been evaluated by simulations and results smaller by $\sim 30-$ $40 \%$, depending on $N_{\text {pad }}$, due to the better defined time profile of the showers.

\section{The blazar Markarian 421}

Mrk421 is one of the brightest blazars and the closest to us $(\mathrm{z}=0.031)$, characterized by a strong broadband flaring activity with a variability time scale ranging from minutes to months. A correlation of VHE gamma rays with X-rays has been observed during single flaring episodes by different detectors and can be interpreted in terms of the Synchrotron Self-Compton (SSC) model, in which X-ray photons are attributed to synchrotron radiation from high energy electrons accelerated in the jet, while VHE photons are due to inverse Compton scattering of the same electrons off the synchrotron photons (Ghisellini et al., 1998).

The Mrk421 high variability makes the long term multiwavelength observation very important to constrain the emission mechanisms models. ARGO-YBJ has monitored Mrk421 for more than 2 years, studying the correlation of the $\mathrm{TeV}$ flux with $\mathrm{X}$-ray data.

\subsection{Gamma ray and X-ray correlation}

In this section we report on the ARGO-YBJ observation of Mrk421 from November 2007 to February 2010, in correlation with the X-ray data of RXTE/ASM $(2-12 \mathrm{keV})$ and

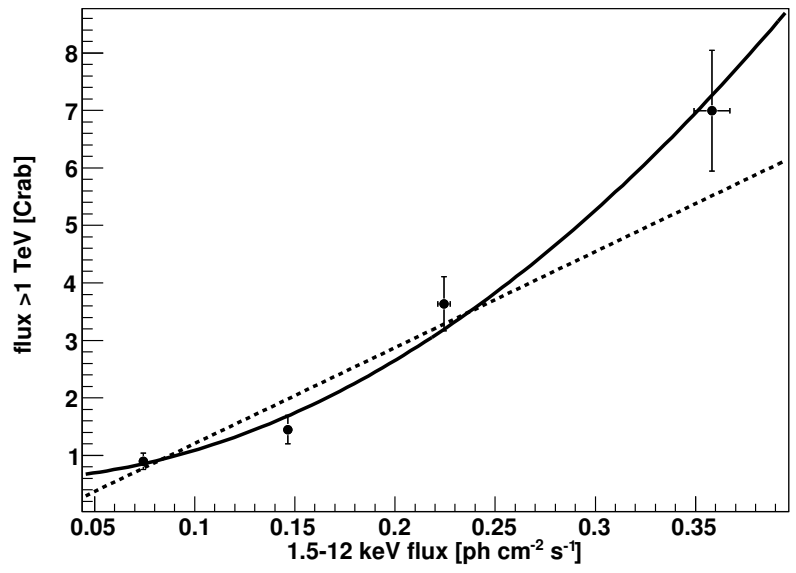

Fig. 2. Mrk421 gamma ray flux $(E>1 \mathrm{TeV})$ vs. X-ray flux $(E=1.5-12 \mathrm{keV})$. The dashed and solid lines are the linear and quadratic fits of the data points, respectively.

Swift/BAT $(15-50 \mathrm{keV})$. The ARGO-YBJ data used in this work consists of showers with $N_{\text {pad }} \geq 60$, corresponding to a photon median energy of $1.1 \mathrm{TeV}$ assuming a power law spectrum with photon index -2.4. A cumulative signal of $\sim 11$ standard deviations has been observed during 676 days.

To investigate the correlation between gamma ray and $\mathrm{X}$ ray emissions, the daily rates obtained by the three detectors have been studied. In total, the data set of simultaneous observations consists of 566 days.

A clear correlation among the rates is visible in Fig. 1, that shows the cumulative light curves obtained by the three experiments. In particular a strong enhancement of the fluxes is clearly seen in the first half of 2008, when the source activity was very high. The flares in June 2008 have been deeply studied by ARGO-YBJ (Aielli et al., 2010) and by a dedicated multiwavelength campaign (Donnarumma et al., 2009).

To quantify the degree of correlation and point out a possible time lag among gamma and X-ray emissions, the discrete correlation function (DCF)(Edelson and Krolik , 1988) has been used. We found that the DCF reaches its maximum value (0.78) for a time lag $T=-0.14 \pm 0.86$ days and $T=-0.94 \pm 1.07$ days, for ARGO-RXTE and ARGO-Swift respectively, consistent with no time lag.

To study the correlation between the $\mathrm{TeV}$ and X-ray fluxes, the data have been divided in 4 groups, according to the X-ray rate measured by RXTE/ASM. Studying the average energy spectra for each group for ARGO-YBJ and RXTE/ASM, we found that the correlation between gammaray and X-ray fluxes is consistent with a quadratic rather than linear relation (see Fig. 2).

Moreover, the data of both detectors show an evident positive correlation between the flux and the spectrum hardness. In particular the photon index of the ARGO-YBJ spectra increases from $-2.48 \pm 0.22$ to $-1.87 \pm 0.21$ when the flux increases from 0.9 to $7 \mathrm{Crab}$ units for $E>1 \mathrm{TeV}$, in agree- 
ment with a previous measurement of the Whipple experiment (Krennrich et al., 2002), suggesting that this is a long term property of the source.

\section{MGRO J1908+06}

The gamma ray source MGRO J1908+06 was discovered by the MILAGRO air shower detector (Abdo et al., 2007) at a median energy of $\sim 20 \mathrm{TeV}$ and recently associated with the Fermi pulsar 0FGL J1907.5+0602 (Abdo et al., 2009). The data were consistent both with a point source and with an extended source of diameter $<2.6^{\circ}$.

The Cherenkov telescope HESS confirmed the discovery with the detection of the extended source HESS J1908+063 (Aharonian et al., 2009) at energies above $300 \mathrm{GeV}$, positionally consistent with the MILAGRO source. The extension of the source, evaluated assuming a symmetrical twodimensional Gaussian shape, was $\sigma_{\mathrm{ext}}=0.34_{-0.03}^{\circ}$.

HESS reported a power law differential energy spectrum with a photon index of $-2.10 \pm 0.07_{\text {stat }} \pm 0.2_{\text {sys }}$ in the energy range $0.3-20 \mathrm{TeV}$, and a differential flux at $1 \mathrm{TeV}$ of $\left(4.14 \pm 0.32_{\text {stat }} \pm 0.83_{\text {sys }}\right) \times 10^{-12} \mathrm{TeV}^{-1} \mathrm{~cm}^{-2} \mathrm{~s}^{-1}$.

More recently, MILAGRO evaluated the energy spectrum of the source (Smith et al., 2009) reporting a power law with an exponential cutoff at high energies. The best fit obtained is $d N / d E=0.62 \times 10^{-11} E^{-1.50} \exp (-E / 14.1)$ $\mathrm{TeV}^{-1} \mathrm{~cm}^{-2} \mathrm{~s}^{-1}$, where $E$ is the energy in TeV. This flux is in disagreement with the one by HESS at a level of 2-3 standard deviations, being about a factor 3 higher at $10 \mathrm{TeV}$.

In the following we present the results of the observation of MGRO J1908+06 by ARGO-YBJ.

\subsection{Determination of the extension and spectrum}

At the ARGO-YBJ site, MGRO J1908+06 culminates at the relatively low zenith angle of $24^{\circ}$ and is visible for $5.4 \mathrm{hrs}$ per day with a zenith angle less than $45^{\circ}$. The data set used in this analysis contains all the showers with zenith angle less than $45^{\circ}$ and $N_{\text {pad }} \geq 40$ recorded in the period from November 2007 to March 2010, for a total of 730.5 days.

The analysis method is the standard search for point gamma ray sources (Aielli et al., 2010) with an optimization of the observational window radius due to the extension of the source.

A sky map in celestial coordinates (right ascension and declination) with $0.1^{\circ} \times 0.1^{\circ}$ bin size, centered on the source position, is filled with the detected events. The cosmic ray background is estimated using the time swapping method (Alexandreas et al., 1993) and subtracted from the event map. Finally the map obtained is "smoothed" to point out the signal, using a circular window with a radius whose value depends on the detector PSF and on the source extension.

To evaluate the extension we assume for simplicity a source shape described by a symmetrical bidimensional

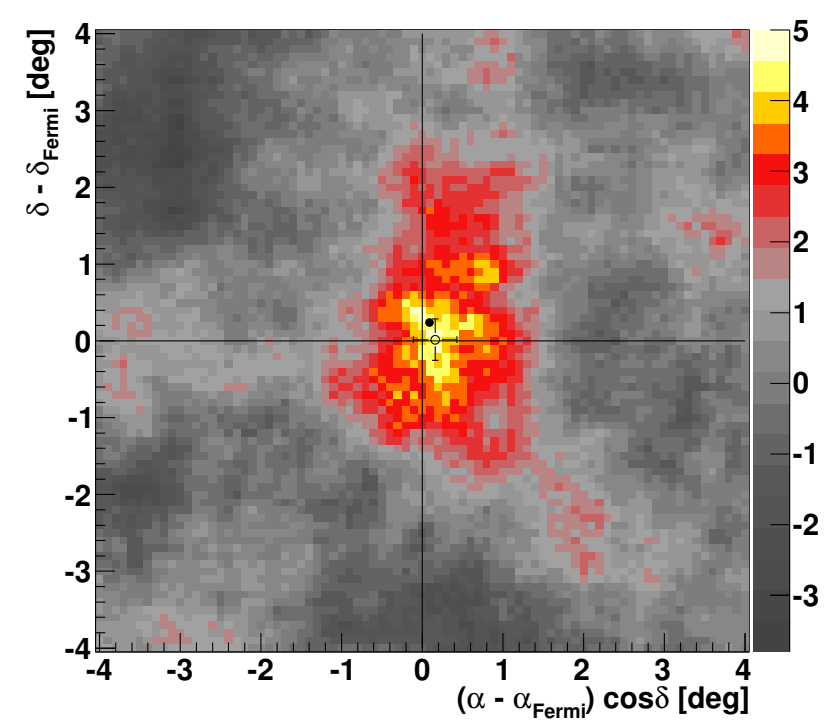

Fig. 3. Significance map of the $8^{\circ} \times 8^{\circ}$ region around MGROJ1908+06 obtained by ARGO-YBJ, for events with $N_{\text {pad }} \geq 100$. The center of the map represents the position of the Fermi pulsar 0FGL J1907.5+0602 $\left(\alpha=286.89^{\circ}, \delta=6.03^{\circ}\right)$. The open and solid circles show the positions measured by MILAGRO and HESS, respectively. The map is smoothed over a circular area of radius $1.1^{\circ}$ (see text).

Gaussian function with r.m.s $=\sigma_{\text {ext }}$, independent of the gamma ray energy.

Fitting the event distribution as a funcion of the distance from the center (set to the Fermi pulsar position) and taking into account the PSF, we found $\sigma_{\mathrm{ext}}=0.48_{-0.28}^{\circ}$, a value larger but consistent with the HESS measurement.

Using the measured extension, we calculate via simulations the optimal window size in order to get the best signal to noise ratio. Fig. 3 shows the significance map for events with $N_{\text {pad }} \geq 100$ of the MGRO1908+06 region. The events of the map have been smoothed with a circular window of radius $1.1^{\circ}$.

An area of excesses with statistical significance $>4$ standard deviations is present near the location of the Fermi pulsar, closer to the positions reported by MILAGRO and HESS. The maximum significance in the region is 4.7 standard deviations. The spatial distribution of the excesses suggests a non symmetrical shape, that needs to be studied in more detail with higher statistics.

For the spectrum determination we assume a simple power law: $d N / d E=K E^{-\gamma}$, without cutoff.

To study the spectral behaviour we define $4 N_{\text {pad }}$ intervals: 40-99, 100-299, 300-999 and > 1000. Then we compare the rate observed in each interval with that given by a simulation assuming a set of test spectra.

The simulation describes the shower development in the atmosphere using the CORSIKA code (Heck et al., 1998), the detector response with a code based on the GEANT pack- 


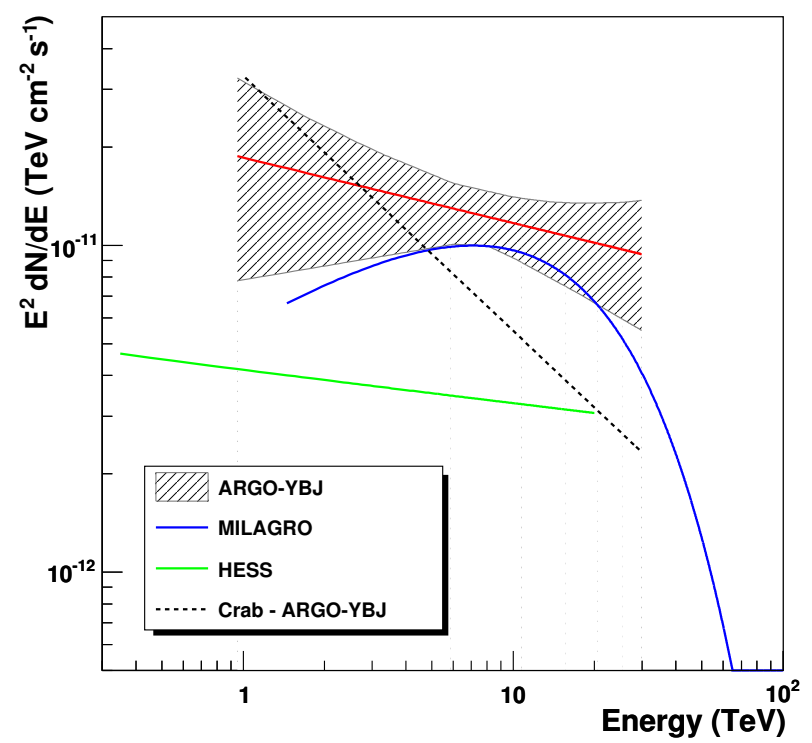

Fig. 4. Gamma ray differential flux from MGRO J1908+06 measured by ARGO-YBJ (red line), multiplied by $E^{2}$. The shaded band represents 1 standard deviation error. The blue and green lines show the fluxes measured by MILAGRO and HESS, respectively. The black dashed line represents the Crab Nebula flux.

age (GEANT, 1993), and finally reconstructs the arrival directions of the showers using the same code used in the data analysis. The reliability of this procedure has been tested studying the Moon shadow deficit (Di Sciascio et al., 2008) and the Crab Nebula and Mrk421 signals (Aielli et al., 2010).

The best fit power law spectrum is: $d N / d E=3.6_{-0.8}^{+0.7}$ $\times 10^{-13}(E / 6 \mathrm{TeV})^{-2.20_{-0.29}^{+0.34}}$ photons $\mathrm{cm}^{-2} \mathrm{~s}^{-1} \mathrm{TeV}^{-1}$ (the errors of the parameters are statistical).

The systematic errors are mainly related to the background evaluation and to the uncertainty in the absolute energy scale. According to our estimate, they globally affect the quoted fluxes for $\lesssim 30 \%$.

To check the existence of a cutoff, we fit the data with a power law spectrum multiplied by the exponential factor $\exp \left(E / E_{\text {cut }}\right)$, varying $E_{\text {cut }}$ from 1 to $50 \mathrm{TeV}$. Decreasing the value of $E_{\text {cut }}$, the best fit gives a spectrum with an increasingly flatter slope, to compensate the loss of events due to the cutoff. However the value of the $\chi^{2}$ remains minimum for a spectrum with no cutoff.

The spectrum found is shown in Fig. 4, together with the spectra by MILAGRO and HESS. The Crab Nebula spectrum (Aielli et al., 2010) is also reported for comparison. Given the flat slope, above a few TeV the MGROJ1908+06 flux becomes larger than the Crab Nebula one.

A significant disagreement is present between the ARGOYBJ and HESS spectra. The flux measured by ARGOYBJ at $6 \mathrm{TeV}$ is $\sim 4$ times larger than the one measured by HESS, while the ARGO-YBJ and MILAGRO data are con- sistent, taking into account the statistical errors (the error of the MILAGRO flux is $\sim 30 \%$ at $10 \mathrm{TeV}$ and larger at lower energies).

One possible cause of the discrepancy among the measurements is that ARGO-YBJ and MILAGRO integrate the signal over a solid angle larger than the HESS one, and are likely to detect more of the diffuse lateral tail of the extended source.

A further interesting possibility is the variability of the source, since the HESS data consist of $27 \mathrm{~h}$ of observations in 2005 - 2007, before our measurements. Further observations are necessary to draw a firm conclusion.

Edited by: J. Poutanen

Reviewed by: two anonymous referees

\section{References}

Abdo, A. A., Allen, B. T., Berley, D. et al.: TeV gamma-ray sources from a survey of the Galactic Plane with Milagro, Astrophys. J. Lett., 664, L91-L94, 2007.

Abdo, A. A., Allen, B. T., Aune, T. et al.: Milagro observations of multi-TeV emission from galactic sources in the Fermi Bright source List, Astrophys. J. Lett., 700, L127-L131, 2009.

Aharonian, F., Akhperjanian, A. G., Anton, G. et al.: Detection of very high energy radiation from HESS J1908+063 confirms the Milagro unidentified source MGRO J1908+06, Astron. Astrophys., 499, 723, 2009.

Aielli, G., Assiro, R., Bacci, C. et al.: Layout and performance of RPCs used in the Argo-YBJ experiment, Nucl. Instr. Meth. A, 562, 92-96, 2006.

Aielli, G., Bacci., C., Bartoli, B. et al.: Gamma-ray flares from Mrk421 in 2008 observed with the ARGO-YBJ detector, Astrophys. J. Lett., 714, L208-L212, 2010.

Alexandreas, D. E., Berley, D., Biller, S. et al: Point source search techniques in ultra high energy gamma ray astronomy, Nucl. Instr. Meth. A, 328, 570-577, 1993.

Di Sciascio, G., on behalf of the ARGO-YBJ collaboration: Physics results from the ARGO-YBJ experiment: Workshop on Frontier object in astrophysics and particle physics, Vulcano, Italy, 2008.

Donnarumma, I., Vittorini, V., Vercellone, S. et al.: The June 2008 flare of Mrk421 from optical to TeV energies, Astrophys. J., 691, L13-L19, 2009.

Edelson, R. R. and Krolik, H. J.: The Discrete Correlation Function, Astrophys. J., 333, 646, 1988.

GEANT - Detector Description and Simulation Tool 1993, CERN Program Library, W5013, 1993.

Ghisellini, G., Celotti, A., Fossati, G. et al.: A theoretical unifying view of gamma-ray bright blazars, Monthly Notices Royal Astron. Soc., 301, 451, 1998.

Heck, D., Knapp, J., Capdevielle, J. N., Shatz, G., and Thouw, T.: Corsika, Forschungszentrum Karlsruhe Rep., FZKA 6019, 1998.

Krennrich, F., Bond, I. H., Bradbury, S. M. et al.: Discovery of spectral variability of Markarian 421 at $\mathrm{TeV}$ energies, Astrophys. J., 575, L9-L13, 2002.

Smith, A. J. for the Milagro Collaboration: A survey of Fermi Catalog sources using data from the Milagro gamma-ray observatory, Proc. Fermi Symp., Washington, D.C, USA, Nov 2-5, 2009. 